\section{BIOSPHERE SOUNDSCAPES}

Leah Barclay, Queensland

Conservatorium of Music, Griffith

University. Brisbane, Australia. Email:

<info@leahbarclay.com>.

See $<$ www.mitpressjournals.org/toc/leon/47/5> for supplemental files associated with this issue.

\section{Abstract}

Biosphere Soundscapes (BioScapes) is a large-scale interdisciplinary art project underpinned by the creative possibilities of soundscape ecology, a rapidly evolving field of biology used to record environmental patterns and changes. This project is designed to inspire communities across the world to listen to the environment and re-imagine the potential of International UNESCO Biosphere Reserves as learning laboratories for a sustainable future.

\section{Introduction}

The global ecological crisis has become a catalyst for interdisciplinary collaborations at a time when a shift in thinking is urgently required. World leaders are now looking towards the validity and possibilities of creative methodologies as tools for change. This presents both a challenge and an unprecedented opportunity for creative practitioners to gain a critical understanding of the situation and devise new processes for a sustainable future. There is an urgent need to listen to the state of our environment and facilitate a sense of interconnection within communities globally. 'Sound' as a catalyst and creative medium is undoubtedly one of the most powerful means to stimulate this shift in consciousness.

In a recent edition of Musicworks, Joel Chadabe stated that the current artistic practices of electroacoustic composers are rooted in the idea that new technologies, unlike traditional musical instruments, can produce sounds used to communicate core messages, including information about the state of our environment. He claims that we are all participating in the emergence of a new type of music accessible to anyone, which can be used to communicate ideas that relate more closely to life than those communicated through traditional musical forms. He believes we need to think of ourselves as "leaders in a magnificent revolution rather than the defenders of an isolate and besieged avant-garde" [1].

Biosphere Soundscapes is a largescale interdisciplinary project underpinned by the creative possibilities of soundscape ecology, a rapidly evolving field of biology used to record environmental patterns and changes. This project is designed to inspire communities across the world to listen to the environment and re-imagine the potential of
UNESCO Biosphere Reserves as learning laboratories for a sustainable future. This project is a key outcome from my practice-led doctoral research that involved conceiving and delivering seven original electroacoustic projects for dissemination in multi-platform environments. The divergent projects were created in cultural immersion, spanning from ambitious sonic explorations in the center of the Amazon Jungle to sounding the rivers of the world through India, Korea, China, Australia and New Zealand. The delivery and dissemination of each project was underpinned by a rich methodology that pivots on a sitespecific project embedded in community cultural engagement.

Throughout these projects it became evident that the environmental interconnectedness many of us have been seeking is still prevalent in a profusion of first nation cultures globally. These ancient knowledge systems argue that the process of simply listening to the environment can completely shift our perception. These collaborative processes transformed my approach to listening and undeniably influenced my creative responses to the environment. My doctoral research began as an exploration of the sustainability of electroacoustic music and evolved into a complex web of projects harnessing electroacoustic music as a change agent. The beginning was fueled by an isolated intention, grounded in a visually dominant western society. Yet through the process of cultural immersion I discovered a tool that provides not only a gratified language of creative expression, but also a voice for the communities and environments collaborating on these projects. The discoveries and observations from each individual project showed a clear trajectory towards a set of tools to initiate cultural changes through environmental electroacoustic music. As a result, the Sonic Ecologies Framework was developed as a means to create an accessible methodology for artists interested in implementing similar projects.

\section{Sonic Ecologies Framework}

The Sonic Ecologies Framework pivots on a site-specific soundscape project embedded in a multi-layered community cultural engagement process developed in response to a specific community. The site specificity requires that this methodology be intrinsically flexible in order to be adaptable within a diversity of environments and communities. It is in essence a practice-led creative research process, taking an ecological approach to contextualising a project within an environment. While there is an essential degree of freedom and adaptability, the process is grounded within the theoretical contexts generated by the artists who experiment and innovate within a continual spiraling between theory and practice.

The Sonic Ecologies framework involves five essential elements: sitespecific subject matter, multi-platform dissemination, community engagement, interdisciplinary partnerships and a longterm strategic vision. While one core creative outcome is most likely the central intention, the adaptability of the project for a range of environments is essential. The research outcomes proved the sonic material should be disseminated widely for maximum exposure; this includes harnessing the power and value of virtual platforms to facilitate global accessibility. The community engagement tools will always evolve depending on the nature and accessibility of the proposed community but the most successful tools throughout this research included: community sound walks, participatory field recording sessions, capacity building workshops and providing access to the appropriate technology for the community to remain engaged in the process.

The Biosphere Soundscapes project was conceived and designed with the Sonic Ecologies framework with a particular focus on the interdisciplinary partnerships resulting from this doctoral research. The artist implementing the Sonic Ecologies framework is initiating a process within a community. The creative outcomes serve as significant milestones but ultimately it is the process that will continue to resonate and evolve over time. As with any form of community engagement, it requires time in order to facilitate change. The capacity building community engagement is designed to empower the community to continue working long after the artist has departed. It is therefore essential that the artist invests critical thought into the methods in which the community will continue to engage and that appropriate technology is accessible to continue working. The most successful strategy resulting from this research was to leave low-cost digital recorders with a key stakeholder in the community and design a web platform to enable the community to continue creating and uploading content. This web platform ignited the beginning of the Biosphere Soundscapes project and 
illustrates the design of a major international project using the Sonic Ecologies framework.

\section{Bioscapes Structure}

Biosphere Soundscapes [2] pivots on a network of site-specific electroacoustic music projects embedded in multilayered community engagement processes within global Biosphere Reserves. This evolving process is implemented by sound artists, acting as agents of change spiraling between contextualised theory and practice. The content generated is embedded in a virtual network of global Biosphere Reserves via google earth technology and shared though the BioScapes community on the website (http://www.biospheresoundscapes.org). The process and creative outcomes are delivered by a core team of artists and advisors who are ultimately acting as catalysts for a global participatory environmental project accessible to anyone with an internet connection.

Composed of 610 sites in 117 countries, Biosphere Reserves are sites recognised under UNESCO's Man and the Biosphere (MAB) programme to promote sustainable development based on local community efforts and sound science [3]. As places that seek to reunite the conservation of biological and cultural diversity and nurture the relationship between people and nature, they are ideal to test and demonstrate innovative approaches to sustainability. Biosphere Reserves also represent a fascinating tool for international collaborations through sharing knowledge, exchanging experiences and building capacity for interdisciplinary collaborations and partnerships. This is the first major international project connecting the soundscapes of global Biosphere Reserves.

The methodological grounding for Biosphere Soundscapes revolves around the creative possibilities of Soundscape Ecology, a rapidly evolving field of biology where scientists record a given habitat and listen for patterns and changes to form an analysis of the health of the environment. The process of working with each Biosphere modifies depending on the collaborating artists and accessibility of the local community. In some instances the process involves sound labs, artist residencies and extensive community engagement, while in other cases the key community stakeholders of the Biosphere Reserve can generate content independently and engage via the website.

\section{Dissemination Platforms}

The virtual platform, developed in collaboration with Australian cultural development agency Feral Arts, is built with three key systems in mind. The first is the BioScapes Residencies, the core activity in implementing this global project. All of the sound, text and imagery from the BioScapes Residencies is geolocated in the interactive Biosphere map and also available through a timeline feature to trace the history of the project and compare the soundscapes. The second system is the BioScapes Lab, which is a more specific structure with research questions revolving around an environmental issue within the proposed Biosphere Reserve. The final system is the BioScapes Community, an accessible platform for anyone to generate content, download educational resources and engage in the global possibilities of the project.

The first two systems are curated platforms, while the accessibility of the BioScapes Community provides realtime interaction and engagement for anyone with internet access. The three systems combine in the Biosphere Soundscapes map, which is a constantly evolving interface that will call attention to our changing sonic environments. The digital platforms will ultimately enable Biosphere networked performance, live streaming tools and the ability to mix soundscapes in real time. This will also provide access to soundscapes currently at risk, allowing virtual collaborators infinite possibilities to explore the sounds of central Australia, the Amazon Jungle or Kenya's Mount Elgon all within an accessible interface.

Biosphere Soundscapes was conceived in 2011 and received concept development funding from Arts Queensland (Regional Arts Development Fund) to deliver a pilot project in the Noosa Biosphere Reserve. The first research lab for Biosphere Soundscapes launched on World Listening Day 2012 (July 18) with an interactive field recording lab in the Noosa Everglades, a forum of international sound artists and a pilot sound map. The project has since involved multiple research labs and the development of specific interfaces and apps for global community engagement and collaboration. There are currently three international residencies in development in Australia and ten Biosphere Soundscapes labs under development across five continents.

Biosphere Soundscapes, although still in preliminary stages of development, highlights the potential role composers and sound artists could play in ecological crisis. In our current state of ecological uncertainty, this project showcases the value of listening as a tool for environmental awareness, community empowerment and cultural change.

Biosphere Soundscapes, along with other creative ventures such as BalanceUnbalance, underpin the true value of UNESCO Biosphere Reserves in bringing passionate communities together to inspire positive change. Biosphere Soundscapes combines art, science, technology and community to give Biosphere Reserves across the world a voice and a global audience to listen.

\section{References}

* This article is based on a paper presented at the 3rd Balance-Unbalance International Conference, 31 May-2 June 2013, Noosa, Queensland, Australia.

1. Joel Chadabe: "A call for avant-garde composers to make their work known to a larger public," in Musicworks, edited by Micheline Roi (Toronto: Musicworks Society of Ontario Inc. 2011) 111: p. 6.

2. Biosphere Soundscapes (online)

$<$ http://www.biospheresoundscapes.org $>$

(visited 15 February 2013)

3. Biosphere Reserves - Learning Sites for Sustainable Development (online):

$<$ http://www.unesco.org/new/en/naturalsciences/environment/ecologicalsciences/biosphere-reserves/> (visited 11 February 2013). 